\title{
INVARIABILITY, ORBITS AND FUZZY ATTRACTORS
}

\author{
S. Perez-Gonzaga, M. Lloret-Climent and J.A. Nescolarde-Selva*. \\ Department of Applied Mathematics. University of Alicante. Alicante. Spain.
}

*Corresponding author: email: josue.selva@ua.es, Telephone. 0034680418381 , Fax number. 0034965909707

\begin{abstract}
In this paper we present a generalization of a new systemic approach to abstract fuzzy systems (Lloret, Nescolarde-Selva \& Perez-Gonzaga, 2014). Using a fuzzy relations structure will retain the information provided by degrees of membership. In addition, to better suit the situation to be modelled, it is advisable to use T-norm or T-conorm distinct from the minimum and maximum respectively. This gain in generality is due to the completeness of the work on a higher level of abstraction. You cannot always reproduce the results obtained previously, and also sometimes different definitions with different views are obtained.In/any case this approach proves to be much more effective when modelling reality.
\end{abstract}

Keywords: fuzzy attractors, fuzzy relations, fuzzy set, invariability, orbits.

\section{INTRODUCTION}

George Cantor (1874) created the foundations of set theory and this was later formalized by Gottlob Frege. Shortly after the publication of this theory Bertrand Russell and Ernst Zermelo discovered the paradox known as Russell's antinomy highlighting the difficulty of the task begun by Cantor and Frege. To resolve these paradoxes different axiomatic set theories were proposed such as the Zermelo-Fraenkel, the Neumann-Bernays-Gödel and the Morse-Kelley on which to build rigorous mathematical theories.

Despite the rich mathematical legacy based on classical logic and axiomatic set theory, many problems in disciplines like engineering, medicine or social sciences retain associated uncertainty. This has lead in recent times to the development of new theories capable of dealing with uncertainty naturally. Fuzzy mathematics born with the theory of fuzzy sets Zadeh (1962) is a focus for this work. Other variants are the theory of rough sets (Pawlak, 1982) or intuitionistic fuzzy set theory Atanassov (1986) which is a generalization of Zadeh's theory of fuzzy sets. Later, Molodtsov (1999) introduced the notion of soft set to study the uncertainty of nonparametric shapes and new developments in this field came from Kharal and Ahmad (2011) who defined applications on soft sets and Bayramov and Gunduz (2013) who introduced important properties of soft topological spaces.

When studying complex problems it is often essential to outline the hierarchy of objects to be studied. Tazaki and Amagasa (1979) proposed a method for modelling the hierarchical structure of some problems in organizational management, it is called fuzzy structural modelling (FSM). The method describes a formal procedure for constructing a hierarchical diagram based on the relationship of each element with the whole. The study of hierarchy has become essential in many fields of science, engineering and sociology. Raghuvanshi and Kumar (1999) generalized the FSM allowing the inclusion of loops of any order. 
One of the main applications of fuzzy theory is to aid decision making. Specifically, Yamashita (1997) proposes a model based on a combination of modeling fuzzy reasoning and FSM to help students who are going to decide what university courses to choose for their careers. There are also many applications of fuzzy logic in medical diagnostics (Dogantekin, 2009; Ansari, 2012) to help medical professionals in their clinical practice. In addition, fuzzy methods have been proposed to help with multi-criteria decisions based on vague sets' theory (Chen and Tan 1994; Hong and Choi 2000) and on intuitionistic fuzzy set theory (Liu and Wang, 2007). Ye (2010) proposed a fuzzy decision making method based on weighted correlation coefficients in intuitionistic fuzzy environments using weighted entropy. Recently, Ye (2012) established measures based on Euclidean distances and Hamming established measures on intuitionistic fuzzy trapezoidal numbers to solve problems like multi-criteria decision-making.

In constructing a probability distribution from incomplete and imprecise data, the quality and quantity of data may have serious problems in practical applications. Kikuchiand Kronprasert (2012) showed how to build a complete probability distribution from an incomplete one when only some of the probabilities are known approximately.

There are several approaches to the concept of fuzzy graphs. Graph theory is applied to problems in areas such as systems analysis, operations research, economics and transportation. However uncertainty may appear in a problem of graph theory. Blue, Blush, and Puckett (2002) present a taxonomy of fuzzy graphs offering a catalogue of different types of fuzzy graphs taking account of the existence of vertices, edges, connectivity and weight of the edges.

The use of fuzzy logic has expanded massively in recent decades. Zadeh (1999) distinguishes two meanings for the term fuzzy logic, in the restricted sense as an extension of multivariate logics and in the broad sense covering the theory of fuzzy sets. In any field classic sets can be replaced by fuzzy sets. For example through fuzzification arithmetic can be generalized to fuzzy arithmetic, topology to blurred topology, control theory to the theory of fuzzy control, and so on (Zadeh, 1999) The present article is precisely about this, introducing a new fuzzy approach to abstract systems by defining the concepts developed earlier by Esteve and Lloret $(2006 a, 2006 b)$ and using the theory of fuzzy sets. We want to analyze what properties are still met in this general framework and what new properties can be demonstrated.

\section{BASIC CONCEPTS}

The generalization of a new systemic focus to abstract systems arises from two aspects: first one can introduce a structure of fuzzy relations, from which you can define the concepts of fuzzy invariance, orbit and attractor and keeping the information provided about the different degrees of membership of the sets and relationships in question; and on the other hand using the concepts of T-norms and T-conorms (Klement, Mesiar, \& Pap, 2000; Bielawski and Tabor, 2012; Baczyński, 2013; Mas, Monserrat, Ruiz-Aguilera, Torrens, 2015; Liu, 2015 ) as a generalization of the maximum and minimum functions. In this way operations between fuzzy sets will be defined more generally as shown below.

\section{Definition 1}


The intersection of two fuzzy sets $A$ and $B$ is defined as follows:

$C=A \cap B \Leftrightarrow \mu_{C}(x)=\mathrm{T}\left(\mu_{A}(x), \mu_{B}(x)\right) \forall x \in M$, where $\mathrm{T}$ is a T-norm.

\section{Definition 2.}

The union of two fuzzy sets $A$ and $B$ is defined as follows:

$C=A \cup B \Leftrightarrow \mu_{C}(x)=C\left(\mu_{A}(x), \mu_{B}(x)\right) \forall x \in M$, where $C$ is a T-conorm.

Consider the abstract fuzzy system $S=(M, R)$ where $M$ is the set of objects that belong to it, and $\mathrm{R}$ the set of relationships between them. The concept of the relationship between the two elements makes the connection determining the fuzzy influences between them. The binary relation represents a connection or transfer of influences between these efements that can be direct or indirect. Indirect influences are generalized in a way determined by the triangulation norms employed.

\section{Definition 3}

In a fuzzy abstract system $S=(M, R)$, if two elements $x_{1}, x_{2} \in M$ verify that for any fuzzy relation $r \in R \quad \mu_{r}\left(x_{1}, x_{2}\right)=p>0$, we can say $x_{1}$ has a direct influence on $x_{2}$ with a degree $p$ mediating the fuzzy relation $r$.

\section{Definition 4}

Let $S=(M, R)$ be an abstract fuzzy system. We say that a set of objects $\left\{x_{1}, \ldots, x_{n+1}\right\}$ forms a chain of relations of size $\mathrm{n}$ if there exists a set of relation $\left\{r_{1}, \ldots, r_{n-1}\right\} \in R$ such that $\left\{x_{1} r_{1} x_{2}, x_{2} r_{2}\right.$ $\left.x_{3}, \ldots, x_{n} r_{n} x_{n+1}\right\}$.

We will extend the definition of triangular norms to make an argument. Thus, if we have a chain of relationships of size $n$ we can calculate the degree of influence using these extended functions.

\section{Definition 5}

Let $\mathrm{T}$ be a T-norm or a T-conorm defined as $[0,1] \times[0,1] \rightarrow[0,1]$. We can extend the domain of T to $n$ arguments in the following manner:

$\mathrm{T}\left(x_{1}, x_{2, \ldots}, x_{n}\right)=\mathrm{T}\left(x_{1}, \mathrm{~T}\left(x_{2}, T\left(x_{3,} . . T\left(x_{n-1}, x_{n}\right) \ldots\right)\right.\right.$

An indirect influence is an influence of an object $x_{1}$ on another object $x_{k+1}$ through another series of objects that act as mediators, that is, there is a chain of relations that begin with $x_{1}$ 
and end with $x_{k+1}$. The size of this chain of relations we will call the step of the influence. The step of the influence denotes the number of binary relations needed to relate $x_{1}$ and $x_{k+1}$.

\section{Definition 6}

Let $S=(M, R)$ be an abstract fuzzy system and the T-norm T: such that $x_{1} \in M$ influences the element indirectly $x_{k+1} \in M$ with a step $k>1$ and degree $p$, if there exists a set of relations $\left\{r_{1}, \ldots\right.$, $\left.r_{r}\right\} \in R$ such that $\left\{\begin{array}{llllllllll}x_{1} & r_{1} & x_{2}, & x_{2} & r_{2} & x_{3}, \ldots, & x_{k} & r_{k} & x_{k+1}\end{array}\right\}$ and verifying that $\mathrm{T}\left(\mu_{r 1}\left(x_{1}, x_{2}\right), \mu_{r 2}\left(x_{2}, x_{3}\right), \ldots, \mu_{r k}\left(x_{k}, x_{k+1}\right)\right)=p$.

\section{STRUCTURE OF FUZZY RELATIONS}

The structure of relationships is the key concept in this theory determining the behaviour of the system. In the previous work by the authors (Lloret et al,2014), this structure of relationships to an object or set of fuzzy objects applied and a non-fuzzy set was obtained. Due to the defuzzification that the structure of relations produces, we lose the information about the degree of relationship between domain objects and the path of the application. The loss of such information is compensated for by being able to explore and reproduce definitions and results that work in the non-fuzzy case and have been demonstrated in other studies (Esteve and Lloret 2006a, 2006b). However, here we embark on a different path concerning the structure of fuzzy relations where, although the theory becomes more complex, the information given about the degree of influence between objects will be preserved. In this way we obtain results that represent reality more faithfully.

We will generalize, therefore, the structure of relationships so that when applied to an element or a fuzzy set, we obtain a fuzzy set.

\section{Definition 7}

Let $S=(M, R)$ be an abstract system and given a fuzzy set $F \subseteq M$, the structure of fuzzy relations applied to the fuzzy set $\mathrm{F}$ is defined as:

$$
\varphi(F)=\left\{\left(\mathrm{y}, \mu_{\varphi}(\mathrm{y})\right): \forall \mathrm{y} \in M\right\} \text { donde } \mu_{\varphi}(\mathrm{y})=\mathrm{C}_{\mathrm{x} \in \operatorname{supp}(\mathrm{F})}\left\{\mathrm{T}_{\mathrm{r} \in \mathrm{R}}\left(\mu_{r}(\mathrm{x}, \mathrm{y}), \mu_{F}(\mathrm{x})\right)\right\}
$$

Where $\mathrm{C}$ is a T-conorm and T a T-norm.

\section{Note 1}

$\left.C_{x \in \operatorname{supp}(F)\left\{T_{r} \in R\right.}\left(\mu_{r}(x, y), \mu_{F}(x)\right)\right\}$ represents the application of the T-conorm $C$ with as many arguments as the cardinal of supp(F) and where each of which is determined by applying the T-norm T with card $(R)+1$ arguments. 
In the case of focussing on a particular relation $r \in R$, the definition will have the following form:

\section{Definition 8}

Let $S=(M, R)$ be an abstract system and given a fuzzy set $F \subseteq M$ and a fuzzy relation $r \in R$ the structure of the relations of the fuzzy set associated to $r$ and applied to the fuzzy set $F$ as:

$\varphi_{r}(F)=\left\{\left(y, \mu_{\varphi}(\mathrm{y})\right): \forall \mathrm{y} \in M\right\}$ where $\mu_{\varphi}(\mathrm{y})=\mathrm{C}_{\mathrm{x} \in \operatorname{supp}(\mathrm{F})}\left\{\mathrm{T}\left(\mu_{r}(\mathrm{x}, \mathrm{y}), \mu_{\mathrm{F}}(\mathrm{x})\right)\right\}$

Where $\mathrm{C}$ is a T-conorm and $\mathrm{T}$ a T-norm.

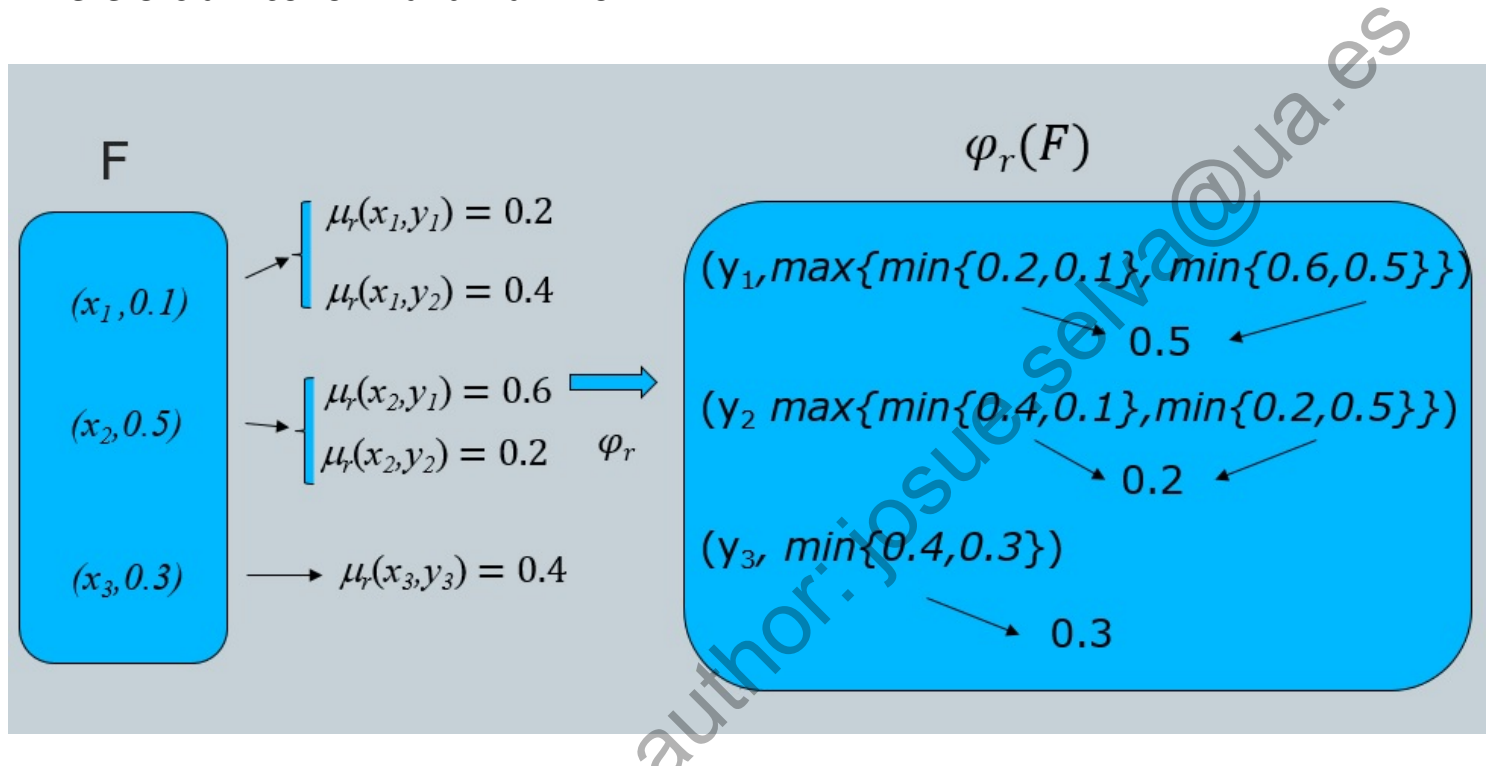

Figure 1. Structure of fuzzy relations on a fuzzy set for triangular norms maximum and minimum

\section{Proposition 1}

Let $S=(M, R)$ be an abstract system with fuzzy subsets $A$ y $B$ of $M$ such that $A \subseteq B$ and let $r \in R$ we have $\varphi_{\mathrm{r}}(A) \subseteq \varphi_{\mathrm{r}}(B)$

\section{Proof}

Let $\mathrm{y} \in M$ with $\mu_{\operatorname{\varphi r}(\mathrm{A})}(\mathrm{y})=\mathrm{C}_{\mathrm{x} \in \operatorname{supp}(\mathrm{A})}\left\{\mathrm{T}\left(\mu_{r}(\mathrm{x}, \mathrm{y}), \mu_{A}(\mathrm{x})\right)\right\}>0$

As $A \subseteq B: \mu_{A}(\mathrm{x}) \leq \mu_{B}(\mathrm{x})$ and as both $\mathrm{T}$ and $\mathrm{C}$ increase monotonically (being triangular norms) we have $C_{x \in \operatorname{supp}(A)}\left\{T\left(\mu_{r}(x, y), \mu_{A}(x)\right)\right\} \leq C_{x \in \operatorname{supp}(A)}\left\{T\left(\mu_{r}(x, y), \mu_{B}(x)\right)\right\}$ Now as $\operatorname{supp}(A) \subseteq \operatorname{supp}(B)$ are $\left\{x_{1}, \ldots x_{n}\right\}=\operatorname{supp}(B) \backslash \operatorname{supp}(A)$ y we call $T_{i=} T\left(\mu_{r}\left(x_{i}, y\right), \mu_{B}\left(x_{i}\right)\right)$ as $i=1 . . n$ As $T$ is one $T-$ norm $0 \leq T_{i} \leq 1$. The 
T-conorm $C_{x \in \operatorname{supp}(B)}\left\{T\left(\mu_{r}(x, y), \mu_{B}(x)\right)\right\}$ will have $n$ arguments more than $C_{x \in \operatorname{supp}(A)}\left\{T\left(\mu_{r}(x, y)\right.\right.$, $\left.\left.\mu_{B}(x)\right)\right\}$. As 0 is the neutral element of the T-conorms $C_{x \in \operatorname{supp}(A)}\left\{T\left(\mu_{r}(x, y), \mu_{B}(x)\right)\right\}$ does not change if one adds $n$ zeros as an argument. If instead of $n$ zeros we put $n T_{i} \geq 0$ as $C$ is increasing monotonically we have: $C_{x \in \operatorname{supp}(A)}\left\{T\left(\mu_{r}(x, y), \mu_{B}(x)\right)\right\} \leq C_{x \in \operatorname{supp}(B)}\left\{T\left(\mu_{r}(x, y), \mu_{B}(x)\right)\right\}$.

Therefore $\forall y \in M: \mu_{\varphi r(\mathrm{~A})}(\mathrm{y}) \leq \mu_{\varphi \mathrm{r}(\mathrm{B})}(\mathrm{y}) \quad \rightarrow \varphi_{\mathrm{r}}(A) \subseteq \varphi_{\mathrm{r}}(B)$

\section{Proposition 2}

Let $S=(M, R)$ be an abstract system with $A$ and $B$ fuzzy subsets of $M$ such that $\operatorname{supp}(A) \cap$ $\operatorname{supp}(B)=\varnothing$ and let $r \in R$ we have: $\varphi_{r}(A \cup B)=\varphi_{r}(A) \cup \varphi_{r}(B)$

\section{Proof.}

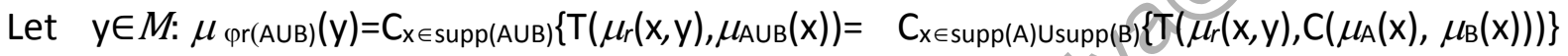
(for the property: $\operatorname{supp}(A \cup B)=\operatorname{supp}(A) \cup \operatorname{supp}(B)$ and the definition of the union) $=\mathrm{C}\left(C_{\mathrm{x} \in \operatorname{supp}(A)}\right.$ $\left\{\mathrm{T}\left(\mu_{r}(\mathrm{x}, \mathrm{y}), \mathrm{C}\left(\mu_{\mathrm{A}}(\mathrm{x}), \mu_{\mathrm{B}}(\mathrm{x})\right)\right)\right\}, \mathrm{C}_{\mathrm{x} \in \operatorname{supp}(\mathrm{B})}\left\{\mathrm{T}\left(\mu_{r}(\mathrm{x}, \mathrm{y}), \mathrm{C}\left(\mu_{\mathrm{A}}(\mathrm{x}), \mu_{\mathrm{B}}(\mathrm{x})\right)\right)\right)=($ Let $\operatorname{supp}(A) \cap \operatorname{supp}(B)=\varnothing)=$ $\mathrm{C}\left(\mathrm{C}_{\mathrm{x} \in \operatorname{supp}(\mathrm{A})}\left\{\mathrm{T}\left(\mu_{r}(\mathrm{x}, \mathrm{y}), \mathrm{C}\left(\mu_{\mathrm{A}}(\mathrm{x}), 0\right)\right)\right\}, \mathrm{C}_{\mathrm{x} \in \operatorname{supp}(\mathrm{B})}\left\{\mathrm{T}\left(\mu_{r}(\mathrm{x}, \mathrm{y}), \mathrm{C}\left(0, \mu_{\mathrm{B}}(\mathrm{x})\right)\right)\right\}\right)=($ let 0 be the neutral element in a T-conorm) $C\left(C_{x \in \operatorname{supp}(A)}\left\{T\left(\mu_{r}(x, y), \mu_{A}(x)\right)\right\}, C_{x \in \operatorname{supp}(B)}\left\{T\left(\mu_{r}(x, y), \mu_{B}(x)\right)\right)=C\left(\mu_{\operatorname{cor}(A)}(y)\right.\right.$, $\left.\mu_{\operatorname{pr}(B)}(\mathrm{y})\right)$ therefore $\varphi_{\mathrm{r}}(A \cup B) \subseteq \varphi_{\mathrm{r}}(A) \cup \varphi_{\mathrm{r}}(B)$

\section{FUZZY INVARIABILITY}

This property is fundamental in the study of a system in the long term and the abundance of invariant sets in a system is a sign of its tendency to behave in a stable manner (Esteve \& Lloret 2006a, 2006b). Using the structure of fuzzy relations, naturally we can define the property of fuzzy invariability.

\section{Definition 9}

Let $S=(M, R)$ be an abstract system and let $A$ be a fuzzy subset of $M$. We say that $\operatorname{supp}(A)$ is invariant if $f_{M}(\operatorname{supp}(A)) \subseteq \operatorname{supp}(A)$.

\section{Definition 10}

Let $S=(M, R)$ be an abstract system. Given $A$ a fuzzy subset of $M$ and a fuzzy relation $r \in R$. We say that $A$ satisfies the property of fuzzy invariability for $\mathrm{r}$ if $\varphi_{\mathrm{r}}(A) \subseteq A$ 


\section{Proposition 3}

Fuzzy invariability implies invariability for the product T-norm and the minimum T-norm.

\section{Proof}

Let $S=(M, R)$ be an abstract system. Given $A$ a fuzzy subset of $M$ and a fuzzy relation $r \in R$. We suppose that $\varphi_{r}(A) \subseteq A$, then $\forall y \in M$ it must be that $\mu_{\operatorname{\varphi r}(\mathrm{A})}(\mathrm{y})=\mathrm{C}_{\mathrm{x} \in \operatorname{supp}(\mathrm{A})}\left\{\mathrm{T}\left(\mu_{r}(\mathrm{x}, \mathrm{y}), \mu_{A}(\mathrm{x})\right)\right\} \leq$ $\mu_{\mathrm{A}}(\mathrm{y})$ We see that $f_{M}(\sup p(A)) \subseteq \operatorname{supp}(A)$. Let $\mathrm{y} \in f_{M}(\operatorname{supp}(A))$ therefore $\exists x \in \operatorname{supp}(A) \mu_{r}(x, y)>0$ y $\mu_{A}(x)>0$ by definition: Therefore $T\left(\mu_{r}(x, y), \mu_{A}(x)\right)>0$ for the minimum and product T-norms $0<\mathrm{T}\left(\mu_{r}(\mathrm{x}, \mathrm{y}), \quad \mu_{A}(\mathrm{x})\right) \quad \leq^{*} \quad \mathrm{C}_{\mathrm{x} \in \operatorname{supp}(\mathrm{A})}\left\{\mathrm{T}\left(\mu_{r}(\mathrm{x}, \mathrm{y}), \quad \mu_{\mathrm{A}}(\mathrm{x})\right)\right\} \leq \mu_{\mathrm{A}}(\mathrm{y}) \rightarrow \mathrm{y} \in \operatorname{supp}(\mathrm{A})$

*For monotony

\section{Proposition 4}

Fuzzy invariability does not imply invariability for drastic T-norms.

\section{Proof}

The drastic T-norm is the smallest of all the T-norms and is defined thus: $\mathrm{T}(a, b)= \begin{cases}a & \text { if } b=1 \\ b & \text { if } a=1 \\ 0 \text { in other cases }\end{cases}$

Let $S=(M, R)$ with $M=\{a, b, c\}$. Let $A \subseteq M$ so that $\sup p(A)=\{a, b\}$ with $\mu_{A}(a)=0.3 \mu_{A}(b)=0.5$. and let $\mathrm{r} \in R$ with $\mu_{r}(\mathrm{~b}, c)=0.7$ y $\mu_{r}(x, y)=0 \forall(\mathrm{x}, \mathrm{y}) \in \operatorname{MxM}(\mathrm{x}, \mathrm{y}) \neq(\mathrm{b}, \mathrm{c})$. It holds trivially that $\varphi_{\mathrm{r}}(A) \subseteq A$ means that $\forall y \in \operatorname{supp}(A) \mu_{\operatorname{\varphi r}(A)}(y)=C_{x \in \operatorname{supp}(A)}\left\{T\left(\mu_{r}(y, x), \mu_{A}(a)\right)\right\}=0$ (For all degrees of membership less than one, the drastic T-norm is worth 0 ) and therefore $\varphi_{r}(A)=\emptyset \subseteq$ A fulfilling fuzzy invariability. On the other hand it is not the case that $f_{M}(\operatorname{supp}(A)) \subseteq \operatorname{supp}(A)$ as $f_{M}(\{a, b\})=$ $c \notin \operatorname{supp}(A)=\{\mathrm{a}, \mathrm{b}\}$ which does not meet invariability

\section{Note 2}

Invariability does not imply fuzzy invariability in general as is shown in the following example.

\section{Example 1}


Let $S=(M, R)$ with $M=\{a, b, c\}$. The fuzzy set $A$ is such that $\operatorname{supp}(A)=\{a, b, c\}$ with $\mu_{A}(a)=0.5$ $\mu_{A}(b)=0.2 \mu_{A}(c)=0.3 \mu_{r}(a, b)=0.7$ y $\mu_{r}(x, y)=0 \forall(x, y) \in M x M(x, y) \neq(b, c)$. $A$ is invariable as $f_{M}(\operatorname{supp}(A)) \subseteq \operatorname{supp}(A): f_{M}(\{a, b, c\})=\{b\} \subseteq \operatorname{supp}(A)=\{a, b\}$. However, fuzzy invariance fails as

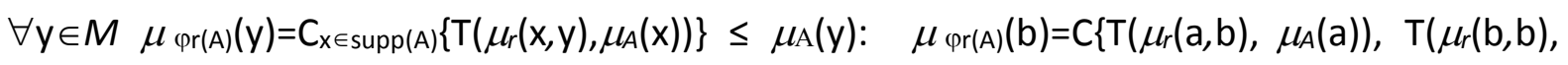
$\left.\left.\mu_{A}(\mathrm{~b})\right), \mathrm{T}\left(\mu_{r}(\mathrm{c}, \mathrm{b}), \mu_{A}(\mathrm{c})\right)\right\}=\mathrm{C}\{\mathrm{T}(0.7,0.5), 0,0\}^{*}=\mathrm{T}(0.7,0.5)=$ (taking the $\mathrm{T}$-norm minimum $)=$ $\min \{0.7,0.5\}=0.5>\mu_{\mathrm{A}}(\mathrm{b})=0.2$.

*0 is the neutral element in a T-conorm

\section{Proposition 5}

Let $S=(M, R)$ be an abstract system. Given $A$ a fuzzy subset of $M$ and a fuzzy relation $r \in R$. If $A$ satisifies fuzzy invariability for $r$, then $\operatorname{\varphi r}(A)$ is also the case.

Proof

Given $A$ the fuzzy invariability $\operatorname{\varphi r}_{r}(A) \subseteq A$ y and applying proposition 1 we have: $\varphi_{r}\left(\varphi_{r}(A)\right) \subseteq \varphi_{r}(A)$

\section{FUZZY ORBITS}

The concept of orbit is the union of the sets that are formed to iteratively apply the structure of relations on an element $\mathrm{x}$ or a given set $\mathrm{A}$. In other words, the orbit includes all elements that are influenced directly or indirectly by $x$ or A respectively (Esteve and Lloret, 2006a, 2006b). Then we define the concept of orbit in our framework of fuzzy set theory and using recently defined structure fuzzy relations.

\section{Definición 11}

Let $S=(M, R)$ be an abstract system, given a fuzzy subset $A$ of $M$ and a fuzzy relation $\mathrm{r} \in R$, we will call the obit of $A$ :

$\operatorname{Orb}_{\mathrm{r}}(A)=\bigcup_{i=0}^{\infty} \mathrm{O}_{\mathrm{i}}(A)$

Being $\mathrm{O}_{0}(A)=A$ y $\mathrm{O}_{n+1}(A)=\varphi_{r}\left(\mathrm{O}_{n}(A)\right)$ with $\varphi_{r}$ the structure of fuzzy relations for $r$. 


\section{Note 3}

Each $\mathrm{O}_{i}$ is a fuzzy set, like its own orbit, formed by the fuzzy union (with the corresponding Tconorm) of these $\mathrm{O}_{i}$

\section{Proposition 6}

Let $S=(M, R)$ be an abstract system and $A$ a fuzzy subset of $M$ and a fuzzy relation $r \in R$. Then $\operatorname{Orb}_{\mathrm{r}}(A)$ is a set that meets fuzzy invariability.

Proof.

We see that $\varphi_{\mathrm{r}}\left(\operatorname{Orb}_{\mathrm{r}}(A)\right) \subseteq \operatorname{Orb}_{\mathrm{r}}(A)$

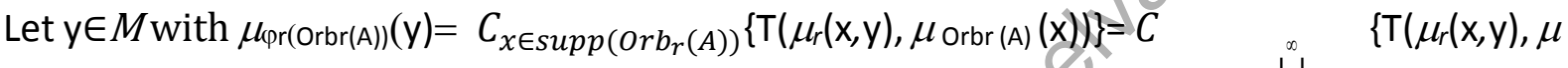
$\mathrm{x} \in \operatorname{supp}\left(\bigcup_{j=0}^{\infty} \mathrm{Oj}\right)$

$\operatorname{Orbr}(\mathrm{A})(\mathrm{x}))\}=C \underset{\mathrm{x} \in \bigcup_{j=0}^{\infty} \operatorname{supp}(\mathrm{Oj})}{ }\left\{\mathrm{T}\left(\mu_{r}(\mathrm{x}, \mathrm{y}), \mu \operatorname{Orbr}(\mathrm{A})(\mathrm{x})\right)\right\}=$

$=\mathrm{C}\left(C_{\mathrm{x} \in \operatorname{supp}\left(\mathrm{O}_{0}\right)}\left\{\mathrm{T}\left(\mu_{r}(\mathrm{x}, \mathrm{y}), \mu \operatorname{Orbr}(\mathrm{A})(\mathrm{x})\right), C_{\mathrm{x} \in \operatorname{supp}\left(\mathrm{O}_{1}\right) \backslash \operatorname{supp}\left(\mathrm{O}_{0}\right)}\left\{\mathrm{T}\left(\mu_{r}(\mathrm{x}, \mathrm{y}), \mu \operatorname{Orbr}\right.\right.\right.\right.$ (A) $\left.\left.(\mathrm{x})\right), \ldots\right\} \leq$ $\mathrm{C}\left(C_{\mathrm{x} \in \operatorname{supp}\left(\mathrm{O}_{0}\right)}\left\{\mathrm{T}\left(\mu_{r}(\mathrm{x}, \mathrm{y}), \mu_{\operatorname{Orbr}(\mathrm{A})}(\mathrm{x})\right), C_{\mathrm{x} \in \operatorname{supp}\left(\mathrm{O}_{1}\right)}\left\{\mathrm{T}\left(\mu_{r}(\mathrm{x}, \mathrm{y}), \mu_{\operatorname{Orbr}(\mathrm{A})}(\mathrm{x})\right), \ldots\right\}\right.\right.$

$=\mathrm{C}\left(\mu_{\varphi_{r}\left(\mathrm{O}_{0}\right)}(y), \mu_{\varphi_{r}\left(\mathrm{O}_{1}\right)}(y), ..\right)=\mathrm{C}\left(\mu_{\mathrm{O}_{1}}(y), \mu_{\mathrm{O}_{2}}(y), ..\right) \leq \mathrm{C}\left(\mu_{\mathrm{O}_{0}}(y), \mu_{\mathrm{O}_{1}}(y), \mu_{\mathrm{O}_{2}}(y), ..\right)=$

$=\mu_{\infty} \quad(y)=\mu_{\mathrm{Orbr}}(\mathrm{A})(y)$ Therefore $\operatorname{\varphi r}_{\mathrm{r}}\left(\operatorname{Orb}_{\mathrm{r}}(A)\right) \subseteq \operatorname{Orb}_{\mathrm{r}}(A)$

$\bigcup_{j=0} \mathrm{Oj}$

\section{Definition 12}

Let $S=(M, R)$ be an abstract system and let $A, B$ be fuzzy subsets of $M$.

We say that $B \neq \varnothing$ is in the orbit of $A$ if $B \subseteq \operatorname{Orb}_{r}(A)$ (i.e. $\mu_{B}(\mathrm{x}) \leq \mu_{\mathrm{Orbr}(\mathrm{A})}(\mathrm{x}) \forall \mathrm{x} \in M$ )

\section{Proposition 7}

Let $S=(M, R)$ be an abstract system and let $A, B$ be fuzzy subsets of $M$ and a fuzzy relation $r \in R$. If $A$ is in the orbit of $B$ for $r$, then $\operatorname{Orb}_{r}(A) \subseteq \operatorname{Orb}_{\mathrm{r}}(B)$ 


\section{Proof}

Beginning with $\mathrm{O}_{0}(A)=A \subseteq \operatorname{Orb}_{\mathrm{r}}(B)$, for proposition $1 \mathrm{O}_{1}(A)=\varphi_{\mathrm{r}}(A) \subseteq \varphi_{\mathrm{r}}\left(\operatorname{Orb}_{\mathrm{r}}(B)\right) \subseteq \operatorname{Orb}_{\mathrm{r}}(B)$ let $\operatorname{Orb}_{\mathrm{r}}(B)$ be an invariant set (proposition 6) Iteratively $\mathrm{O}_{\mathrm{n}}(A) \subseteq \operatorname{Orb}_{\mathrm{r}}(B) \forall \mathrm{n}$ Natural, therefore $\operatorname{Orb}_{\mathrm{r}}(A)=\bigcup_{i=0}^{\infty} \mathrm{O}_{\mathrm{i}}(A) \subseteq \operatorname{Orb}_{\mathrm{r}}(B)$

\section{Proposición 8}

Let $S=(M, R)$ be an abstract system and let $A$ be a fuzzy subset of $M$ and let there be a fuzzy relation $\mathrm{r} \in R$. If $A$ satisfies fuzzy invariability for $r$, therefore $\operatorname{Orb}_{\mathrm{r}}(A) \subseteq A$.

\section{Proof}

$\mathrm{O}_{0}(A)=A \subseteq A$. If $A$ satisfies the property of fuzzy invariability for $\mathrm{r}: \mathrm{O}_{1}(A)=\varphi_{\mathrm{r}}(A) \subseteq A$ applying proposition $1 \mathrm{O}_{2}(A)=\varphi_{\mathrm{r}}\left(\mathrm{O}_{1}(A)\right) \subseteq \varphi_{\mathrm{r}}(A) \subseteq A$, iteratively $\mathrm{O}_{\mathrm{n}}(A) \subseteq A \quad \forall \mathrm{n}$ Natural , therefore $\mathrm{Orb}_{\mathrm{r}}$ $(A)=\bigcup_{i=0}^{\infty} O_{i}(A) \subseteq A$

\section{Corollary 1}

Let $S=(M, R)$ be an abstract system, let $A$ and $B$ be fuzzy subsets of $M$ and let a fuzzy relation $r \in R$ complementing fuzzy invariability in $A$ for $r$ : If $B$ is in the orbit of $A$, then $B \subseteq A$

\section{Proof}

$B \subseteq \operatorname{Orb}_{r}(A)$ and for the anterior proposition $\operatorname{Orb}_{r}(A) \subseteq A$, therefore $B \subseteq A$

\section{FUZZY ATTRACTORS}

In system dynamics an attractor is a set towards which a system tends to evolve when it is in certain spaces. Esteve and Lloret (2007) defined attractors for abstract discrete systems mediating structures and relations. These sets are called attractors and can be found at the end of the orbits of objects and we will show an application in the fuzzy case.

In applying the concept of attractor for the case of an abstract fuzzy system it is interesting to see that the focus given by using the fuzzy relations structure and therefore by iterating obtains fuzzy sets. 


\section{Definition 13}

Let $S=(M, R)$ be an abstract fuzzy system, let there be a fuzzy set $C \subseteq M$ and a fuzzy relation borrosa $r \in R$. Let $A_{0}=\operatorname{supp}(C)$ y $A_{k}=f_{M}\left(\operatorname{supp}\left(A_{k-1}\right)\right) \mathrm{k}=1,2, \ldots$ fulfilling $A_{k+1} \subseteq A_{k} \mathrm{k}=1,2, \ldots$ therefore we define the attractor $A$ as $A=\bigcap_{i=0}^{\infty} A_{k}$ and the set $C$ we will call basin of attraction.

In the case of the above definition, the attractor A would be the subset of M which ends up being confined orbits of any subset $C$.

\section{Example 2}

Let $S=(M, R)$ be a dynamic fuzzy system where: $M=\{a, b, c, d, e, f, g, h, i, j, k, l, m, n\}$ and let the relation $r \in R$ represented in figure 3 by blue arrows showing the degree of the relation. This figure shows an attractor $A$ and a basin of $C$.

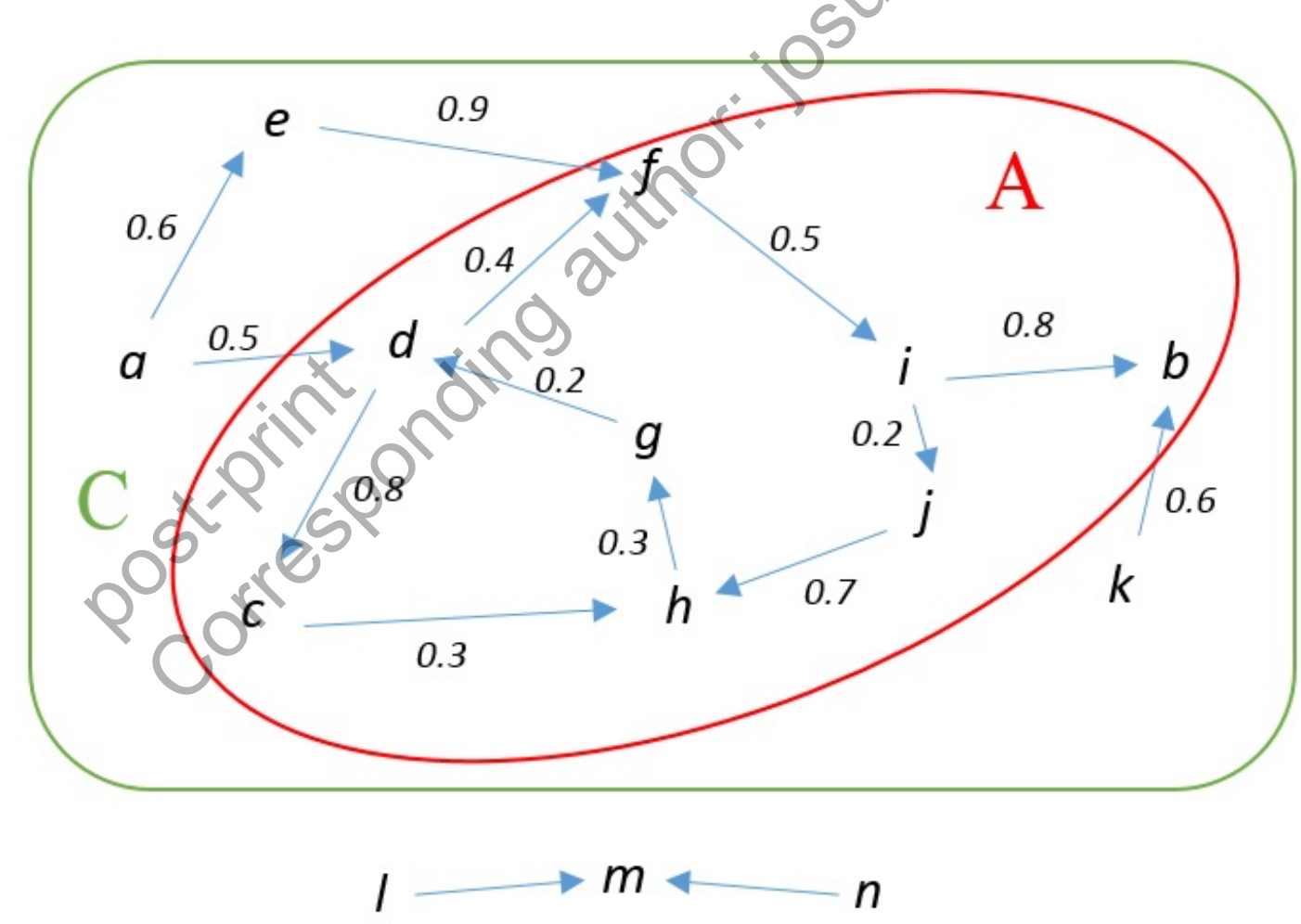

Figure 3. Attractor and basin of attraction of an abstract fuzzy system 


\section{Note 4}

In this example we can see on one hand that for an attractor $A$, the basin of attraction $C$ need not be unique: if from $\mathrm{C}$ we remove the element $\mathrm{k}$, we get another basin of attraction that also generates the attractor $A$. On the other hand, included in the attractor $A$, we can find other attractors : for example considering $A^{\prime}=\{d, g, h, c\}$ we see that $A^{\prime}$ is an attractor based on, for example, the basin of attraction $C^{\prime}=\{d, g, h, c, j\}$.

Using the structure of fuzzy relations we will give the definition of fuzzy attractor.

\section{Definition 14}

Let $S=(M, R)$ be a fuzzy abstract system, let there be a fuzzy set $C \subseteq M$ and a fuzzy relation $\mathrm{r} \in R$. If we define $A_{0}=C$ y $A_{k}=\varphi_{\mathrm{r}}\left(A_{k-1}\right) \mathrm{k}=1,2, \ldots$ Then we define the fuzzy attractor $A$ as $A$ $=\bigcap_{i=0}^{\infty} A_{k}$, where the intersection is defined by a T-norm 5 .

\section{Proposition 9}

Let $S=(M, R)$ be a fuzzy abstract system and let there be a fuzzy set $A \subseteq M$ that is a fuzzy attractor of the system $S$. It is the case that $A$ has fuzzy invariability.

\section{Proof}

Let $A$ be a fuzzy attractor $A=\bigcap_{i=0}^{\infty} A_{k}$ with $A_{0}=C$ y $A_{k}=\varphi_{\mathrm{r}}\left(A_{k-1}\right) \quad \mathrm{k}=1,2, \ldots \quad A=\bigcap_{i=0}^{\infty} A_{k} \rightarrow$ $\varphi_{\mathrm{r}}(A) \subseteq \varphi_{\mathrm{r}}\left(\bigcap_{i=0}^{\infty} A_{k}\right) \smile \bigcap_{i=0}^{\infty} \varphi_{r}\left(A_{k}\right)=\bigcap_{i=1}^{\infty} A_{k}=\bigcap_{i=0}^{\infty} A_{k}=A \quad\left(A_{k}\right.$ is $\quad$ a $\quad$ contractive succession)

\section{CONCLUSION}

In this article we generalize two aspects of the theory introduced in a previous publication (Lloret et al, 2014). On the one hand we introduce the triangular norms that allow us to operate fuzzy sets with more general functions that enable us to obtain more general results where possible. Then specific applications are chosen for the triangular standard which most suits the model presented. Another way of generalization is given by what we have defined as a structure of fuzzy relations, that instead of applying ordinary sets in ordinary sets, we 
apply fuzzy sets in fuzzy sets. The great advantage of this is that information is preserved that is sometimes essential for our models. Based on the concept of the structure of fuzzy relations, we revised the concept of invariability introduced in the second section for another type of invariability: fuzzy invariability. Besides using both the structure of fuzzy relationship sand triangular norms we introduced two new concepts: the orbit and the fuzzy attractor. The latter after first defining the concept of attractor using the structure of relationships.

In addition to introducing these concepts we have provided a number of examples and shown relations between them: We relate invariability with fuzzy invariability, orbits and attractor with invariant sets and fuzzy attractors with fuzzy invariability in a manner that allows us to find ways to identify these special sets. The theory introduced here is applicable to simulate real systems such as systems of beliefs or epistemological arguments, and these are the objectives of future studies.

\section{REFERENCES}

Ansari, A. Q., Gupta, N. K., \& Ekata, E. (2012, November). Automatic Diagnosis of Asthma Using Neurofuzzy System. In Computational Intelligence and Communication Networks (CICN), 2012 Fourth International Conference on(pp. 819-823). IEEE.

Atanassov, K. T. (1986). Intuitionistic fuzzy sets. Fuzzy sets and Systems,20(1), 87-96.

Baczyński, M. (2013). On two distributivity equations for fuzzy implications and continuous, Archimedean t-norms and t-conorms. Fuzzy Sets and Systems. 211, 34-54.

Bayramov, S. \& Gunduz, C. (2013). Some Results on Fuzzy Soft Topological Spaces. Mathematical Problems in Engineering, 2013.

Bielawski, J and Tabor, J. (2012). A t-norm embedding theorem for fuzzy sets. Fuzzy Sets and Systems. 209, 33-53.

Blue, M., Bush, B., \& Puckett, J. (2002). Unified approach to fuzzy graph problems. Fuzzy Sets and Systems, 125(3), 355-368.

Cantor, G. (1874). Uber eine Eigenschaft des Inbegriffes aller reellen algebraischen Zahlen. Journal für die reine und angewandte Mathematik, 77, 258-262.

Chen, S. M., \& Tan, J. M. (1994). Handling multicriteria fuzzy decision-making problems based on vague set theory. Fuzzy sets and Systems, 67(2), 163-172. 
Dogantekin, E., Dogantekin, A., \& Avci, D. (2009). Automatic hepatitis diagnosis system based on linear discriminant analysis and adaptive network based on fuzzy inference system. Expert Systems with Applications, 36(8), 11282-11286.

Esteve-Calvo, P. F., \& Lloret-Climent, M. (2006). Coverage and invariability by structural functions. International Journal of General Systems, 35(6), 699-706.

Esteve-Calvo, P. F., \& Lloret-Climent, M. (2006). Coverage, invariability and orbits by structural functions. Kybernetes, 35(7/8), 1236-1240.

Hong, D. H., \& Choi, C. H. (2000). Multicriteria fuzzy decision-making problems based on vague set theory. Fuzzy sets and systems, 114(1), 103-113

Kharal, A., \& Ahmad, B. (2011). Mappings on soft classes. New Mathematics and Natural Computation, 7(03), 471-481

Kikuchi, S., \& Kronprasert, N. (2012). Effects of data quality and quantity in systems modelling: a case study. International Journal of General Systems,41(7), 697-711.

Klement, E. P., Mesiar, R., \& Pap, E. (2000). Triangular norms. Springer.

Liu, H. W. (2015). Distributivity and conditional distributivity of semi-uninorms over continuous t-conorms and t-norms. Fuzzy Sets and Systems. 268, 27-43.

Liu, H. W., \& Wang, G. J. (2007). Multi-criteria decision-making methods based on intuitionistic fuzzy sets. European Journal of Operational Research, 179(1), 220-233.

Lloret-Climent, M., Nescolarde-Selva, J. A., \& Pérez-Gonzaga, S. (2014). Coverage and invariability in fuzzy systems. International Journal of General Systems, 43(1), 96-104.

Mas, M., Monserrat, M., Ruiz-Aguilera, D., Torrens, J. (2015). Migrative uninorms and nullnorms over t-norms and t-conorms. Fuzzy Sets and Systems. 261, 20-32.

Molodtsov, D. (1999). Soft set theory-first results. Computers \& Mathematics with Applications, 37(4), 19-31.

Pawlak, Z. (1982). Rough sets. International Journal of Computer \& Information Sciences, 11(5), 341-356. 
Raghuvanshi, P. S., \& Kumar, S. (1999). On the structuring of systems with fuzzy relations. Systems, Man, and Cybernetics, Part B: Cybernetics, IEEE Transactions on, 29(4), 547-553.

Tazaki, E., \& Amagasa, M. (1979). Structural modeling in a class of systems using fuzzy sets theory. Fuzzy Sets and Systems, 2(1), 87-103

Yamashita, T. (1997). On a support system for human decision making by the combination of fuzzy reasoning and fuzzy structural modeling. Fuzzy Sets and Systems, 87(3), 257-263.

Ye, J. (2010). Fuzzy decision-making method based on the weighted correlation coefficient under intuitionistic fuzzy environment. European Journal of Operational Research, 205(1), 202-204.

Ye, J. (2012). Multicriteria group decision-making method using the distances-based similarity measures between intuitionistic trapezoidal fuzzy numbers. International Journal of General Systems, 41(7), 729-739.

Zadeh, L. A. (1962). From circuit theory to system theory. Proceedings of the IRE, 50(5), 856865.

Zadeh, L. A. (1999). Fuzzy logic and the calculi of fuzzy rules, fuzzy graphs, and fuzzy probabilities. Computers \& Mathematics with applications, 37(11), 35. 